\title{
Ganzheitliches Kundenbeziehungsmanagement für Finanzdienstleistungen
}

\author{
Robert Winter \\ Institut für Wirtschaftsinformatik, Universität St. Gallen \\ Müller-Friedberg-Strasse 8, 9000 St. Gallen, Schweiz \\ Robert.Winter@unisg.ch
}

\section{Einleitung}

Spätestens seit Mitte der 1980er Jahre konzentriert sich das Marketing nicht mehr nur auf die Gestaltung einzelner Interaktionen, sondern betrachtet zusätzlich oder sogar schwerpunktmässig die Gestaltung der gesamten Kundenbeziehung (z.B. [Dwyer / Schurr / Oh 1987] [Rogers / Peppers 1994] [Peter / Schneider 1994]). Vor der Hintergrund zunehmender Käufermacht in saturierten, transparenten Märkten ist diese Neuorientierung aus heutiger Sicht naheliegend: Einzelne Transaktionen bzw. Interaktionen basieren schliesslich im Normalfall auf einer umfassenderen, längerfristigen Kunden- bzw. Lieferantenbeziehung. Im Gegensatz zum Transaktionsmarketing, das die einzelnen Transaktionen isoliert betrachtet und bei dem Kundenakquisition und Verkauf im Vordergrund stehen, konzentriert sich deshalb das Beziehungsmarketing auf die langfristige Erhaltung der Kundenbeziehung, d.h. die Kundenbindung [Schulze 2000, S.12-13].

Etwa ab Mitte der 1990er Jahre werden Customer Relationship ManagementSysteme (CRM-Systeme) als Standardsoftware angeboten, um Informationen zur Unterstützung der Frontoffice-Prozesse im Marketing, Verkauf und Service zu sammeln, zu analysieren und zu bewerten. CRM-Systeme sollen Kundengewinnung und Kundenbindung verbessern, die Wirtschaftlichkeit erhöhen und die Interaktionsmöglichkeiten mit Kunden verbessern [Schulze 2000, S.18]. Allerdings werden viele Projekte zur Einführung von CRM-Systemen als Misserfolge gewertet, weil z.B. die in der Standardsoftware unterstellte Form von Kundenbeziehungen von der Realität im betreffenden Unternehmen abweicht, weil wichtige Funktionalitäten wie z.B. die ganzheitliche Bewertung von Kundenbeziehungen nicht unterstützt werden oder weil dem bereichs- und funktionsübergreifenden Charakter des Kundenbeziehungsmanagements durch die Software oder ihre Einführung nicht Rechnung getragen wird [Peppart 2000].

Im Finanzdienstleistungsbereich wird der Anwendung von CRM-Systemen ein besonders grosses Potenzial zugemessen: 
- Die Leistungen haben einen hohen Informationsanteil, so dass die durch Kundeninteraktionen erzeugten Datenbestände grosse Potenziale für Informationsgewinnung zum Zweck einer gezielten Marktbearbeitung schaffen.

- Die Leistungen werden ganz oder teilweise elektronisch abgewickelt. Dadurch wird es möglich, Marktbearbeitung und Leistungsabwicklung zumindest in Teilbereichen zu automatisieren und integrieren.

- Zumindest im Retailgeschäft wird eine grosse Zahl von Kundenbeziehungen unterhalten und eine grosse Zahl von Leistungen erbracht. Diese grosse Grundgesamtheit erlaubt die Typisierung von Kundenbeziehungen und die Ableitung (und Verwendung) von Referenzlösungen.

Im Finanzdienstleistungsbereich werden aus diesen Gründen überdurchschittlich steigende Investitionen in CRM getätigt. Gartner erwartet für das Jahr 2004 ein CRM-Marktvolumen von global 1 Milliarde US-Dollar allein für Finanzdienstleistungen [Seeger 2001, S.99-100]. Die Hauptprobleme, die CRM-Projekte im Finanzdienstleistungsbereich nach [Seeger 2001, S.100-101] überwinden müssen, sind das Aufbrechen vertikaler, produktorientierter Abwicklungsstrukturen (sog „Produktsilos"), die Integration von Altapplikationen sowie die Fähigkeit, Projektwirkungen über die Grenzen einzelner Organisationseinheiten hinweg zu quantifizieren und zu kontrollieren.

„Organisations need to understand the theoretical and practical implications of the business perspective of CRM before embarking upon a CRM system project. [...] To be successful, CRM projects need to be viewed as more than the implementation of IT.“ [Light 2001, p.1239] Seit vielen Jahren wird bei der Einführung von insbesondere in Form von Standardsoftware implementierten - Applikationen so vorgegangen, dass zunächst Strategien und Prozesse aus fachlicher Sicht spezifiziert werden, um danach allfällige Anpassungen entweder dieser Grundlagen oder der Applikation vorzunehmen, so dass am Ende Fachkonzept und ITUnterstützung konsistent sind. Im Bereich des Kundenbeziehungsmanagements existieren zwar mittlerweile bewährte IT-Lösungen; Fachliche Grundlagen im Sinne von Beziehungsmanagement-Strategien oder -Prozessen sind jedoch weit weniger expliziert. Dieser Beitrag hat zum Ziel, die fachliche (d.h. konzeptionelle) Perspektive des Kundenbeziehungsmanagements im Finanzdienstleistungsbereich zu rekonstruieren.

Im zweiten Abschnitt werden dazu zunächst als Grundlagen die verschiedenen Typen von CRM-Systemen analysiert, das Architekturmodell für Retail Banking im Informationszeitalter im Hinblick auf Kundenbeziehungsmanagement adaptiert und Vorschläge zum Einführungsprozess von CRM-Systemen untersucht, die Hinweise auf konzeptionelle Grundlage liefern könnten. Im dritten und vierten Abschnitt werden die Potenziale des Kundenbeziehungsmanagements aus Unternehmens- bzw. aus Kundensicht systematisiert. Ein Vorschlag für mögliche konzeptionelle Grundlagen des Kundenbeziehungsmanagements auf Strategie- und Prozessebene findet sich in Abschnitt 5. Dort wird auch ein Ausblick auf die Um- 
setzung durch entsprechende CRM-Systeme gegeben. Der abschliessende sechste Abschnitt fasst die Ergebnisse des Beitrags zusammen und diskutiert mögliche Anschlussfragestellungen wie etwa die Ausdehnung des hier skizzierten, ganzheitlichen Kundenbeziehungsmanagement-Konzepts auf Lieferanten- und andere geschäftliche Partnerbeziehungen.

\section{Grundlagen}

\subsection{Rollen von CRM-Systemen in der Informationssystem- Architektur}

Für die Analyse der konzeptionellen Grundlagen des Kundenbeziehungsmanagements erscheint es sinnvoll, CRM-Systeme nicht hinsichtlich Ihrer (Implementierungs-)Komponenten zu strukturieren (z.B. Prozesse vs. Wissensstruktur vs. Basis [Schmid / Bach / Österle 2000, S. 23]), sondern hinsichtlich der verschiedenen Rollen, die sie in der Informationssystem-Architektur des Unternehmens spielen (teilweise nach [Systor 2001]):

- Operatives CRM: Informationssysteme werden zur möglichst weitgehenden Automatisierung von Kampagnen, von Loyalitätsprogrammen sowie von Vertriebsprozessen genutzt

- Analytisches CRM: Informationssysteme werden zur Unterstützung der Identifikation möglicher Kunden-Produkt-Zuordnungen (sog. „Leads“) genutzt. $\mathrm{Zu}$ den Aufgaben des analytischen CRM gehören auch die Integration von Kundeninformationen über alle Produkte und Kanäle hinweg sowie die Prognose des Kundenverhaltens.

- Kollaboratives CRM: Informationssysteme werden zur Unterstützung der individuellen Kundenansprache, der Erzeugung individualisierter Angebote sowie zur Abwicklung von Kundeninteraktionen unter Nutzung verschiedenster Kanäle genutzt.

- Strategisches CRM: Informationssysteme werden zur Planung, Kontrolle und Überarbeitung von Profitabilitätsanalysen, Segmentierungen, Kanalzuordnungen und Kampagnen benutzt.

Informationssystem-Architekturen aus der Praxis (z.B. [Williams 2000], [Seeger 2001, S.103], [Systor 2001]) umfassen operatives, analytisches und manchmal kollaboratives CRM. Diese Informationssysteme werden häufig als Komponenten eines Feedback-Regelkreises angeordnet (,,closed loop“ [Systor 2001]). Das Fehlen des strategischen CRM in solchen Architekturen stellt - neben den in der Einführung beschriebenen Überlegungen - ein weiteres Indiz für die Notwendigkeit einer intensiveren Betrachtung der konzeptionellen Grundlagen des CRM dar. 
Für die Rekonstruktion der fachlichen Grundlagen des Kundenbeziehungsmanagements wird festgehalten, dass insbesondere die Planung, Kontrolle und Überarbeitung von Profitabilitätsanalysen, Segmentierungen, Kanalzuordnungen und Kampagnen adressiert werden muss.

\subsection{Grundmodell der Architektur des Kundenbeziehungs- managements}

Als Grundlage für die Spezifikation der konzeptionellen Grundlagen des Kundenbeziehungsmanagements wird das vier Hauptebenen umfassende Grundmodell der „Bankenarchitektur des Informationszeitalters“ herangezogen. Dieses Grundmodell wurde in [Fugmann et al. 1999] erstmals in Grundzügen publiziert. Die Modellierungsebenen wurden in [Leist / Winter 1999] sowie [Leist / Winter 2000] ausgeführt und in [Heinrich / Leist 2000] insbesondere im Hinblick auf die fachliche Modellierung detailliert. Das Modell wird in durch Leist ([Leist 2001], Beitrag in diesem Band) erstmals umfassend dargestellt.

Dieses Grundmodell hat sich für die Strukturierung verschiedenster Bereiche des Bankgeschäfts bewährt. Diese Generalität prädestiniert es dafür, auch als Basis für die Gestaltung des Kundenbeziehungsmanagements zu dienen. Seine Adaption führt zu dem folgenden Vorgehensmodell zur Spezifikation des Kundenbeziehungsmanagements im Finanzdienstleistungsbereich:

- Auf der Geschäftsebene ist aus fachlicher Sicht zu spezifizieren, wie Kundenbeziehungen grundsätzlich gestaltet werden sollen und welche Rolle ihnen im jeweiligen Geschäftsmodell zukommt (das ,was“ der fachlichen Modellierung).

- Auf der Prozessebene ist - ebenfalls aus fachlicher Sicht - zu spezifizieren, durch welche Aktivitäten und mit Hilfe welcher Führungsgrössen die im vorherigen Schritt skizzierten Ziel-Kundenbeziehungen geplant, organisiert und gesteuert werden können (das ,wie“ der fachlichen Modellierung).

- Auf der Applikationsebene werden aus Sicht des Informationssystemmanagements die Komponenten des Informationssystems spezifiziert, d.h. die im vorherigen Schritt identifizierten Kundenbeziehungsprozesse sind so auf das Informationssystem abzubilden, dass eine sinnvolle Applikationsstrukturierung entsteht. Falls eine CRM-Applikation bereits in Form eines als Standardsoftware realisierten CRM-Systems vorliegt, ist sie möglichst konsistent mit anderen Applikationen zu integrieren, um eine überlappungs- und lückenfreie Zuordnung zu Kundenbeziehungsprozessen entsteht.

- Auf der Softwareebene sind schliesslich aus Sicht des Informatikmanagements die Applikationsspezifikationen in geeignete Softwaremodule bzw. -komponenten zu transformieren, wobei nicht mehr eine überlappungs- und lückenfreie Gesamtarchitektur, sondern ein optimaler Wiederverwendungs- 
und Integrationsgrad im Vordergrund steht. Falls Softwaremodule oder -komponenten bereits in Form eines als Standardsoftware realisierten CRMSystems vorliegen, sind sie - soweit notwendig - möglichst konsistent mit anderen Softwaremodulen und -komponenten zu integrieren.

\subsection{Vorgehensmodelle für die Strukturierung der Einführung von CRM-Systemen}

Zur Rekonstruktion der konzeptionellen Grundlagen des Kundenbeziehungsmanagements können Vorgehensmodelle für die Einführung von CRMStandardsoftware hilfreich sein. Gerade bei der Einführung von Informationssystemen sehen frühe Phasen ja die Erarbeitung der konzeptionellen Grundlagen und die schrittweise Überführung dieses Fachkonzepts in die Parametrisierung der Standardsoftware vor [Alpar et al. 2000, S.283-295].

Schulze [Schulze 2000a, Kapitel 4] [Schulze 2000b, S.58-67] differenziert strategie-, prozess-, system- und wissensorientierte Ansätze zur CRM-Einführung. Strategieorientierte Ansätze nehmen die Definition einer CRM-Strategie zum Ausgangspunkt, die über kritische CRM-Erfolgsfaktoren und Anforderungsanalysen an das CRM-System die Basis für „business cases“, „business processes“ und „,business objects“ im CRM bildet. Prozessorientierte Ansätze fokussieren auf das kundenorientierte Redesign der Unternehmung, das besonders starke Bezüge zur Organisationsentwicklung und zum Change Management hat. Systemorientierte Ansätze sind stark Standardsoftware-orientiert und konzentrieren sich auf die systematische Einführung des CRM-Systems. Wissensorientierte Ansätze fokussieren auf den sukzessiven Lernprozess, der aus der Integration und Analyse von Kundeninformationen resultiert. Er schlägt einen prozessorientierten Ansatz vor, der mit der Kundenpotenzial- und Kundenprozessanalyse startet, CRM-Prozesse und die entsprechende Prozessführung konzipiert und diese Spezifikationen auf ein entsprechendes CRM-System transformiert [Schulze 2000a, Kapitel 5][Schulze 2000b, S.71-83].

Für die Rekonstruktion konzeptioneller Grundlagen für Kundenbeziehungsmanagement zeigt die Auswertung der durch Schulze untersuchten CRMEinführungsmethoden, dass Aktivitäten wie z.B. Kundeninformationsintegration, Kundenzufriedenheitsanalyse, kundenbedürfnisorientierte Segmentierung, Produkt-Segment-Kanal-Planung, Interaktionskonfiguration [Schulze 2000a, S.65-76] offensichtlich wichtige Bausteine des strategischen CRM darstellen. Allerdings steht die Systematisierung und Explizierung dieser Komponenten noch aus. 


\section{Potenziale des Informationssystem-gestützten Kundenbeziehungsmanagements aus Unternehmenssicht}

Beim Beziehungsmanagement geht es letzlich darum, „persönliche“ Beziehungen zu profitablen Kunden zu knüpfen, zu pflegen und zu nutzen [Schulze 2000a, S.17]. Die Potenziale von CRM-Systemen lassen sich nicht nur nach Unternehmens- oder Kundensicht differenzieren (zur Kundensicht s. nachfolgender Abschnitt); Auch aus Unternehmenssicht lassen sich Potenziale aus technischer Sicht (z.B. Datenintegration, Automatisierung von Marketing- oder Vertriebsprozessen) und Geschäftspotenziale differenzieren. Da die technischen Potenziale letzlich der Realisierung von Geschäftspotenzialen dienen müssen, erfolgt die Beschreibung der Unternehmenssicht auf CRM-Potenziale im Folgenden aus der geschäftlichen Perspektive.

Die Realisierung dieser Potenziale kann aber nicht nur aus technischer Sicht, sondern auch aus organisatorischer Sicht zu abweichenden Strukturierungen führen. So unterscheidet z.B. Reisinger zwischen Potenzialmanagement (z.B. Erkennen von Kundenverhalten, Aufzeigen von Cross-Selling-Potenzialen), Multi-ChannelMarketing (z.B. Unterstützung kanalübergreifender Vertriebsprozesse), Kampagnenmanagement (z.B. optimierte Direkt-Marketing-Massnahmen) und Datenmanagement (z.B. Aufbau einer integrierten Kunden-Datenbasis) [Reisinger 2001, S.16+17]. Im Folgenden wird von der organisatorischen Ausgestaltung des Kundenbeziehungsmanagements abstrahiert; Im Vordergrund soll allein die konzeptionelle Geschäftssicht stehen.

\subsection{Potenziale}

Aus Geschäftssicht lassen sich die folgenden Potenziale des Kundenbeziehungsmanagement unterscheiden:

- Kundengewinnung: Durch Kundenbeziehungsmanagement können prospektive neue Kunden gezielt kontaktiert werden und es können individualisierte und damit attraktive - Angebote gemacht werden, wodurch die Effektivität der Kundengewinnung steigt [Schulze 2000a, S.52-54].

- Verlängerung profitabler Kundenbeziehungen: Kundenbeziehungen durchlaufen verschiedene Phasen, die sich nach anfänglicher Unprofitabilität aufgrund von Akquisitionskosten durch zunehmende Profitabilität aufgrund sinkender Preissensibilität, cross selling und up selling auszeichnen [Schulze 2000a, S.61-63; Swift 2001, p.43]. Profitable Kundenbeziehungen müssen so lange wie möglich profitabel gehalten oder sogar noch profitabler gemacht werden. Kundenbeziehungsmanagement kann dazu beitragen, in dem zusätzliche Verkaufspotenziale identifiziert werden und / oder indem die Abwande- 
rung profitabler Kunden durch „Individualisierung“ der Preisgestaltung, Rabatte o.ä. verhindert wird.

- Umwandlung wenig profitabler Kundenbeziehungen in profitable: Aufgrund hoher Akquisitionskosten, geringer Verweildauer und / oder geringer Verkäufe können Kundenbeziehungen auch nach einiger Zeit unprofitabel oder wenig profitabel sein. Kundenbeziehungsmanagement hilft, solche Kundenbeziehungen zu erkennen und durch cross selling oder up selling profitabel zu machen.

- Erkennung und Beendigung unprofitabler Kundenbeziehungen: Viele Kundenbeziehungen können nicht profitabel werden, weil keine Reaktion auf entsprechende Unternehmensaktivitäten erfolgt. Kundenbeziehungsmanagement kann helfen, solche Kundenbeziehungen zu identifizieren und durch Veränderung von Konditionen, in letzter Konsequenz aber möglicherweise auch verweigerte Vertragsverlängerungen oder Vertragskündigungen zu beenden.

\subsection{Voraussetzungen}

Um die im vorangehenden Abschnitt beschriebenen Geschäftspotenziale des Kundenbeziehungsmanagements nutzen zu können, müssen jedoch bestimmte Voraussetzungen gegeben sein bzw. geschaffen werden:

- Ganzheitliche Sicht auf Kundenbeziehungen: Nicht einzelne Transaktionen, sondern erst deren Aggregation über alle Produkte und Kanäle zum kumulativen Kundenwert (zusammen mit einer Prognose des customer lifetime value) stellen eine geeignete Grundlage für die Bewertung einer Kundenbeziehung dar. Ebenso stellen in vielen Branchen nicht einzelne Kundenbeziehungen, sondern erst deren Aggregation über alle Beziehungen einer Familie, einer Lebensgemeinschaft o.ä. zu einem „Beratungsverbund“ [Reisinger 2001] eine geeignete Grundlage für die Bewertung von Kundenbeziehungen dar. Wird eine so weit wie möglich reichende Aggregation von Transaktionen und Kundenbeziehungen nicht durchgeführt, kann es passieren, dass z.B. ein vermögender Privatkunde wegen der ,schlechten“ Behandlung seines studierenden Kindes als angeblich nicht überziehungskreditwürdiger Bankkunde seine Kundenbeziehung beendet oder dass ein guter Geschäftskunde aufgrund standardisierter Behandlung als Retail-Privatkunde lukrative Geschäfte zu einem Mitbewerber verlagert. Auch der Zeitaspekt spielt bei der Aggregation der relevanten Informationen eine Rolle: Wird kein ausreichend komplexes Ereignis- und Beziehungsmodell für Privatkunden genutzt, kann es z.B. passieren, dass ein/e prospektiver Erbe/in nicht ausreichend „gepflegt“ wird, weil er/sie zur Zeit noch geringes Einkommen oder Vermögen hat. 
- Kosten- und Ertragstransparenz: Die Beschreibung der Potenziale des Kundenbeziehungsmanagements zusammen mit den Ausführungen zur ganzheitlichen Sicht auf Kundenbeziehungen machen deutlich, dass die Profitabilität von Kundenbeziehungen permanent und zeitnah unter Nutzung von Lebenszyklus- und Beziehungsnetzmodellen bewertet werden muss. Voraussetzung für eine solche Bewertung ist höchstmögliche Kosten- und Ertragstransparenz. Je monolithischer das Geschäftsmodell organisiert ist, desto schwieriger wird eine eindeutige Zurechnung von Kosten und Erträgen werden (siehe Beitrag des Autors im ersten Teil dieses Buches).

- Gezielte Marktbearbeitung: Je gezielter die Ansprache potenzieller Kunden hinsichtlich deren aktuellem Produktportfolio, deren aktueller Lebensphase, eventuellen aktuellen Lebensereignissen oder eventuellen aktuellen Transaktionen kontaktiert werden, desto höher ist die Wahrscheinlichkeit erfolgreichen cross sellings, erfolgreichen up sellings oder der erfolgreichen Aufnahme einer Kundenbeziehung. Erst die Aggregation aller verfügbaren Informationen zusammen mit einer zeitnahen Auswertung aktueller Veränderungsindikatoren, einer sehr hohen Zahl von Segmenten und schliesslich einer effektiven Kampagnenunterstützung erlauben eine systematische und gezielte Marktbearbeitung.

- Schaffung von "lock-in": Aufgrund vergleichbarer Professionalitätsstandards des Wettbewerbs, hoher Markttransparenz für Konsumenten und sinkender Kundenloyalität können langdauernde Kundenbeziehungen nahezu ausschliesslich durch effektive Kundenbindungsprogramme geschaffen werden. Erst wenn eine attraktive Kompensation in Form von Prämien, Vergünstigungen oder insbesondere individualisierten Sonderleistungen (z.B. Eintrittsbillets zu exklusiven Sport- oder Kulturveranstaltungen, die den Präferenzen des Kunden entsprechen) gewährt wird, werden attraktive Kunden zu geringerer Preissensitivität, höherer Loyalität, höherer Abhängigkeit und / oder dem Verzicht auf Akquisitionsprämien durch den Wettbewerb bereit sein.

\section{Potenziale des Informationssystem-gestützten Kundenbeziehungsmanagements aus Kundensicht}

Im vorangehenden Abschnitt wurden die Potenziale des Informationssystemgestützten Kundenbeziehungsmanagements aus Unternehmenssicht betrachtet. Allerdings werden entsprechende Aktivitäten nur dann erfolgreich sein, wenn sie auch aus Kundensicht einen Mehrwert gegenüber traditionellen, transaktionszentrierten Vermarktungs- und Vertriebsstrategien erzeugen. Grundsätzlich werden sowohl die Individualisierung von Angeboten bzw. Leistungen wie auch die ganz- 
heitliche Abdeckung von Kundenprozessen von Kunden als Mehrwert wahrgenommen [Winter 2001b].

- Individualisierung von Leistungsangeboten: Je spezifischer ein Angebot auf das aktuelle Produktportfolio, die aktuelle Lebensphase, eventuell eingetretene aktuelle Lebensereignisse oder eventuelle aktuelle Transaktionen eines Kunden eingeht, desto eher wird das Angebot als attraktiv eingeschätzt. Wenn Kundenbeziehungsmanagement Unternehmen in die Lage versetzt, attraktive Angebote zu machen, wird dies auch aus Kundensicht positiv bewertet. So sind z.B. Telekomprovider dazu übergegangen, profitablen Kunden aufgrund ihrer individuellen Telekommunikationsgewohnheiten Tarife anzubieten, die als attraktiv wahrgenommen werden, weil sie vordergründig für häufig vorkommende Verbindungen Rabatte gewähren.

- Ganzheitliche Abdeckung von Bedürfnissen: „Consumers may not use the term, but what they really want is ,aggregation“- one-stop access to all their financial data." [Berinato 2001]. Durch Aggregation von Bankverbindungen, integrierte Planungs- und Auswertungsfunktionen, ein breites Informationsangebot zu Finanzdienstleistungen verschiedener Anbieter und ggf. Community-Funktionen wie Diskussionsforen oder Produktbewertung können Finanzprozesse bei Konsumenten ganzheitlich abgedeckt werden. So integriert z.B. yourhome.ch verschiedenste Finanzdienstleistungen, Informationsdienste, Hilfsmittel (z.B. Entwurfs-, Lokalisierungs- und Bewertungswerkzeuge) und Marktübersichten rund um den Prozess „Wohneigentumserwerb“ in der Schweiz [Schmid / Erni-Grüter / Bach 2001].

- Multikanalfähigkeit: Prognosen gehen davon aus, dass nur jeweils ca. $20 \%$ der Retail-Bankkunden Zugang zu Finanzdienstleistungen ausschliesslich über traditionelle Kanäle („,Filial-Traditionalisten“) oder ausschliesslich über elektronische Kanäle nachfragt; $60 \%$ der Retail-Bankkunden präferieren einen gleichartigen Zugang zu Finanzdienstleistungen über verschiedenste Kanäle, d.h. von der Filiale über Bancomaten und Phonebanking aus Fest- und Mobilnetz bis zu PC- oder PDA-gestütztem electronic Banking [Sparkassenzeitung 2001]. Nur durch multikanalfähiges Kundenbeziehungsmanagement, d.h. die konzeptionelle Trennung von Produktabwicklung, Kanalabwicklung und Kundenbeziehungsmanagement [Winter 2001a] lässt sich sicherstellen, dass Kunden trotz eines Zugangs über verschiedenste Kanäle konsistent behandelt werden und konsistente Angebote bekommen [Gronover / Riempp 2001].

Die Voraussetzungen zur Nutzung dieser Potenziale aus Kundensicht unterscheiden sich deutlich von den Voraussetzungen aus Unternehmenssicht (s. vorangehender Abschnitt). Informationsaggregation und Angebotsindividualisierung finden dort ihre Schranken, wo der Eindruck des "gläsernen Kunden“ entsteht oder wo Informationen über den erlaubten Anwendungsbereich hinaus (z.B. in Unternehmensnetzwerken) oder gar völlig ohne Zustimmung ausgewertet werden. Bei 
der ganzheitlichen Kundenprozessunterstützung muss davon ausgegangen werden, dass - gerade auch aufgrund des hohen Selbstorganisationsgrads der sich dadurch bildenden Communities - echte Auswahl und objektive Beratung erfolgen müssen (d.h. die Möglichkeiten zur Herausstellung eigener Angebote begrenzt sind) und dass ein bestimmter Anteil von Konsumenten und (z.B. sehr spezifischen) Konsumentenprozessen durch vorstrukturierte, ganzheitliche Prozessunterstützung nicht erreicht werden kann oder will.

\section{Konzeptionelle Grundlagen des Kundenbeziehungsmanagements}

\subsection{Geschäftsebene}

Das Geschäftsmodell spezifiziert u.a. die Kernprodukte (in Bezug auf die unterstützten Kundenprozesse), die Art des Vertriebskontakts, die Vertriebspolitik, die Preispolitik, die Markenkonzeption sowie die Abnehmer- und Kundengruppe [Heinrich 2000, S.43-50]. Damit sind ganz wesentliche Grundlagen für die Kundenbeziehungsmanagement-Strategie bereits festgelegt. Innerhalb dieser Grenzen ist nun zu spezifizieren, „was“ das Beziehungsmanagement erzeugen soll (vgl. Beschreibung der Modellierungsebenen in Abschnitt 2.2).

Zunächst müssen die auf Vertrags- bzw. Produktebene vorliegenden, transaktionsorientierten Informationen nicht nur auf die Ebene von Kundenbeziehungen, sondern weiter bis auf die Ebene von Vertriebsverbünden integriert werden. Als Vertriebsverbund wird eine Menge von Kundenbeziehungen definiert, die gemeinsam zu pflegen sind, weil aufgrund von Eigentumsverhältnissen, Familienverhältnissen, Partnerschaften o.ä. Interaktionen mit einer der Kundenbeziehungen die jeweils anderen Kundenbeziehungen beeinflussen. Beispielsweise muss die RetailKundenbeziehung zu einem Mittelständler mit der Geschäftskundenbeziehung des betreffenden Unternehmens verbunden werden, und die Retail-Kundenbeziehung eines Studierenden oder eines prospektiven Erben muss mit der Private BankingBeziehung eines vermögenden Elternteils bzw. Verwandten verbunden werden.

Ist eine integrierte Gesamtsicht der Vertriebsverbünde geschaffen, können diese bewertet werden. Der „Wert“ eines Vertriebsverbunds kann im Sinne eines sog. "lifetime value" beispielsweise als Barwert der jeweils prognostizierten Transaktions-Deckungsbeiträge über die prognostizierte Rest-Lebensdauer der enthaltenen Kundenbeziehungen abgeleitet werden. Oft kehrt dabei die langfristige Betrachtung die traditionelle Kundenbeziehungs-Bewertung um: So entstehen z.B. für Studierende, Individualkunden und vermögenden Älteren bei lifetime-Bewertung völlig andere Bewertungen als bei kurzfristiger Betrachtung [Petzel 2001]. 
Sind die Werte der Vertriebsverbünde bekannt, kann deren Verteilung zur Bildung von Segmenten herangezogen werden. Diesen Segmenten können dann geeignete Massnahmen zugeordnet werden, wenn die jeweils segmentspezifisch prognostizierten Massnahmenerträge die jeweils segmentspezifisch prognostizierten Massnahmenkosten nicht übersteigen.

Im Folgenden wird dieses Vorgehensmodell an einem Beispiel illustriert. In Abbildung 1 werden die erwarteten lifetime values der Vetriebsverbünde auf der horizontalen Achse, die relativen Häufigkeiten der jeweils erwarteten lifetime values auf der vertikalen Achse abgetragen. Aus Vereinfachungsgründen wird eine Normalverteilung der lifetime values zwischen (seltenen) negativen Werten und (ebenso seltenen) extrem hohen Werten angenommen; Die Form der Verteilung spielt für das hier vorgestellte Vorgehensmodell keinerlei Rolle.

Auf Grundlage der Häufigkeitsverteilung der lifetime values werden sechs Segmente gebildet: Segment \#1 umfasst die Vertriebsverbünde mit sehr negativem lifetime value, Segment \#2 solche mit gering negativem lifetime value, Segment \#3 solche mit gering positivem lifetime value, Segment \#4 das Mittelfeld usw.

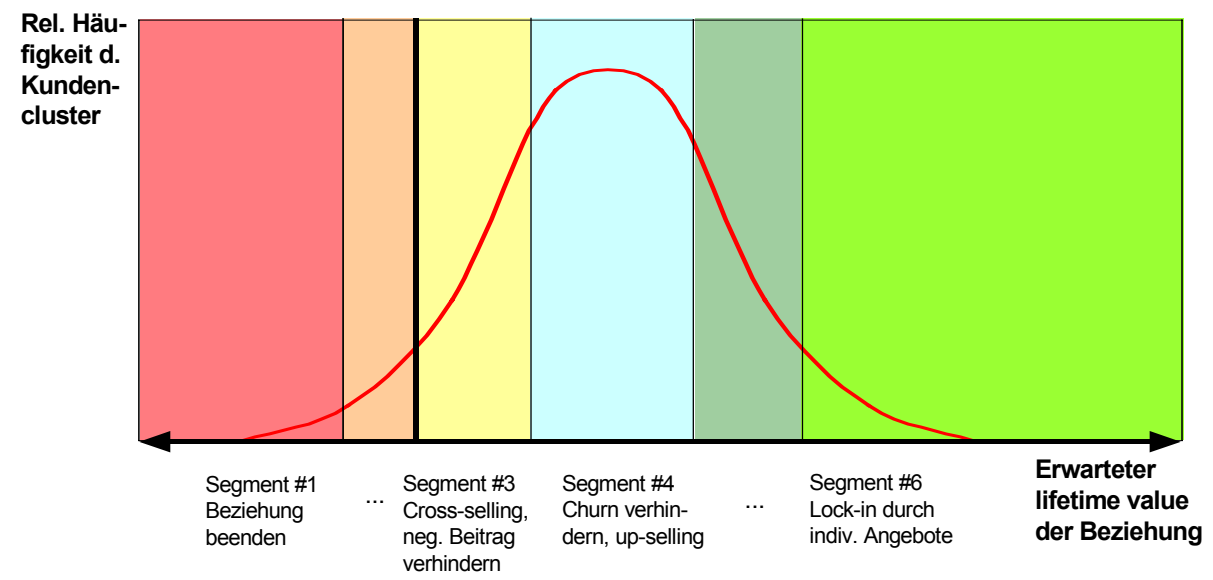

Abbildung 1: Analyse der Häufigkeitsverteilung des lifetime value

Für jedes Segment erfolgt nun eine Analyse, in der für verschiedene Standard-Massnahmen (z.B. Beziehung beenden, up selling, Angebotsindividualisierung) Kosten und Erträge prognostiziert werden.

Abbildung 2 zeigt angenommene Kosten- und Ertragsverläufe für die Segmente \#2 (S1), \#4 (S2) und \#6 (S3). 


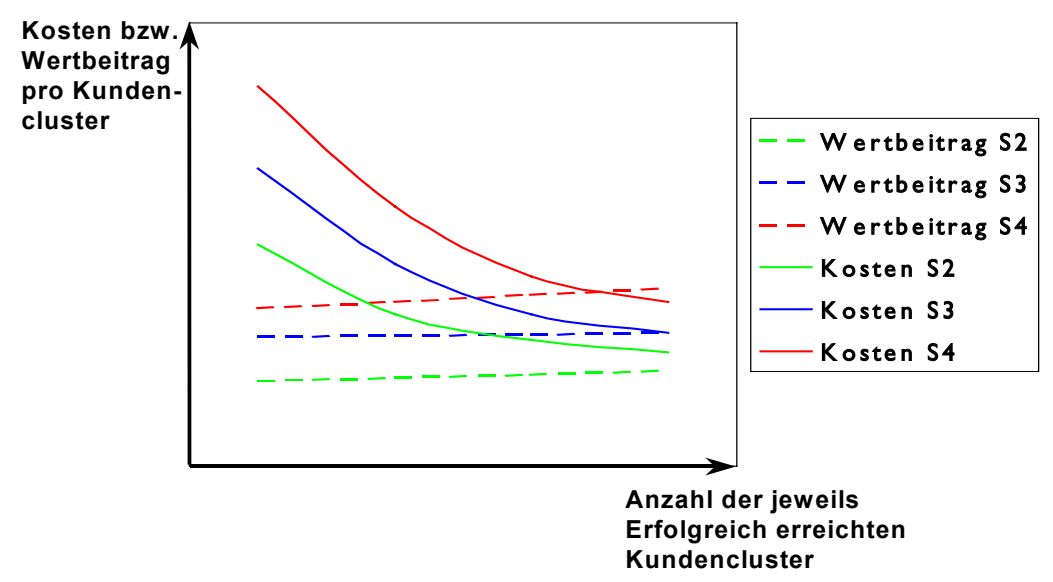

Abbildung 2: Kosten- und Ertragsanalyse für Standard-Massnahmen

Für jede Massnahme in jedem Segment ergibt sich aufgrund der sinkenden Durchschnitts-Massnahmenkosten pro erreichtem Vertriebsverbund und der mindestens konstanten Durchschnitts-Massnahmenerträge pro erreichtem Vertriebsverbund irgendwann ein break even. Die unterschiedliche Grösse der Segmente zusammen mit den jeweiligen Kurvenverläufen determiniert aber, ob die jeweilige Massnahme im betrachteten Segment tatsächlich sinnvoll einsetzbar ist.

Aus der Kombination der jeweils als erfolgversprechend identifizierten Massnahmen ergeben sich für das jeweils betrachtete Segment Normstrategien. In unterschiedlichen Unternehmungen können auf diese Weise für ähnlich definierte Segmente durchaus unterschiedliche Strategien ermittelt werden, da die Zahl der jeweils umfassten Vertriebsverbünde pro Segment und sowie die Kosten- und Ertragsverläufe unternehmensspezifisch sind.

\subsection{Prozessebene}

Auf Prozessebene sind die das „was“ definierenden Ergebnisse der Strategieebene (Normstrategien, z.B. in Segment \#4 Kundenabwanderung verhindern) so zu spezifizieren, dass ihre Umsetzung strukturiert, organisiert und geführt werden kann. 
Als Hauptprozesse des Kundenbeziehungsmanagement werden immer wieder (z.B. [Schmid / Bach / Österle 2000, S.24-26] [Schulze 2000a, S.18-19] [Systor 2001])

- Marketing (Adressat ist der Markt, d.h. insbesondere Nicht-Kunden; Ziel ist die Kundenansprache),

- Verkauf (Adressaten sind Interessenten; Ziel ist der Vertragsabschluss) und

- Service (Adressaten sind Kunden)

genannt. Allerdings fällt es schwer, diese sehr abstrakten Hauptprozesse eindeutig den in konkreten CRM-Beschreibungen genannten Basisprozessen zuzuordnen. So lässt sich z.B. das Kampagnenmanagement sowohl dem Marketing wie auch dem Verkauf zuordnen: Kampagnen können sowohl neue Märkte entwickeln wie auch bestehende Kunden z.B. für cross selling adressieren.

Deshalb wird hier vorgeschlagen, die abstrakte Hauptprozess-Ebene zu überspringen und als oberste Betrachtungsbene der Prozessebene konkrete Kundenbeziehungsmanagement-Referenzprozesse zu wählen. Kandidaten für solche Referenzprozesse sind z.B. Kampagnenmanagement, Kontaktmanagement, Kundenbindungsmanagement, scoring (d.h. Ermittlung des lifetime value), event detection oder Faktorenanalyse (für die Ermittlung von Abwanderungsursachen, „churn detection“). Ausser solchen Referenzprozess-Kandidaten mit Kundenbezug müssen auch noch Unterstützungsprozesse wie z.B. die Profitabilitätsmessung einschliesslich Produkt-, Kanal- und Kontaktpunktrechnung standardisiert werden. Die Strukturierung der Prozesslandkarte für Kundenbeziehungsmanagement stellt eine der wichtigsten Forschungsaufgaben in diesem Bereich dar, da es noch keine allgemein anerkannten Referenzprozesse gibt, wie sie z.B. in ERP-Systemen zu finden sind.

Neben Referenzprozessen im engeren Sinne sind auf der Prozessebene auch konzeptionelle Informationsmodelle für die Repräsentation von Vertriebsverbünden, Lebensereignissen, Kontakt- bzw. Transaktions-Lebenszyklen sowie Zusammenhängen zwischen Transaktionen, Kontakten und Selling-Potenzialen zu spezifizieren. Auch hier gibt es Vorschläge (z.B. ein einfaches KundenLebensphasenmodell in [Swift 2000]), die aber ebenfalls noch nicht allgemein akzeptiert oder validiert sind.

Auf der Grundlage ausreichend detaillierter, auf die jeweilige Unternehmung angepasster Referenzprozesse und einer geeigneten Anpassung der skizzierten Referenzmodelle kann dann eine systematische Zuordnung von (Standard-)Applikationenskomponenten erfolgen. Basieren CRM-Systemeinführungen auf solchen Grundlagen, darf erwartet werden, dass die eingangs beschriebenen, aus dem Fehlen konzeptioneller Überlegungen resultierenden Probleme in CRMProjekten nicht oder zumindest nur in abgeschwächter Form auftreten. 


\section{Zusammenfassung und Ausblick}

In diesem Beitrag wurde versucht, die „business perspective“, d.h. die nicht ITspezifischen Grundlagen für die Einführung von CRM-Systemen zu identifizieren. Grundlage der Überlegungen war neben einem generellen Architekturmodell die Analyse von CRM-Systemarchitekturen und CRM-Einführungsmethoden. Auf dieser Grundlage wurden als wesentliche Potenziale des Informationssystemgestützten Kundenbeziehungsmanagement aus Unternehmenssicht eine rationalere „Bewirtschaftung“ von Kundenbeziehungen und aus Kundensicht Individualisierung, Ganzheitlichkeit und Kanalunabhängigkeit identifiziert. Die Nutzbarkeit dieser Potenziale erfordert jedoch aus Unternehmenssicht eine fortschrittliche Informationslogistik, eine fortgeschrittene Organisation sowie geeignete Strategien zur Sicherung der Kundenloyalität. Aus Kundensicht sind die informationelle Selbstbestimmung, die unvollständige Standardisierbarkeit komplexer Kundenprozesse und die sinkende Bereitschaft, sich fest an bestimmte Anbieter zu binden, zu beachten.

Auf Strategieebene wurde eine Methode zur Ermittlung segmentspezifischer Normstrategien für das Kundenbeziehungsmanagements skizziert. Die Suche nach Kundenbeziehungsmanagement-Referenzprozessen und -Referenzmodellen machte deutlich, dass allgemein akzeptierte Prozesse und Modelle fehlen. Dies gilt insbesondere für die Repräsentation von Vertriebsverbünden (d.h. Kundenclustern), Lebensereignissen, Kontakt- und Transaktions-Lebenszyklen sowie Zusammenhängen zwischen Transaktionen, Kontakten und Selling-Potenzialen. Auch das Fehlen einer standardisierten Methode zur Profitabilitätsmessung von Kundenbeziehungsmanagement-Massnahmen verhindert eine bessere Absicherung von CRM-Einführungsprojekten und CRM-Dauerbetrieb.

Obwohl die in diesem Beitrag vorgestellten Konzepte selbstverständlich noch weiterer Operationalisierung und vor allem Validierung bedürfen, stellen sie einen ersten Schritt $\mathrm{zu}$ einem „konzeptionellen Fundament“ für CRMEinführungsprojekte dar.

Allerdings besteht weiterhin das generelle Problem, dass vernetzte Geschäftsarchitekturen und zunehmende Virtualisierung von Wertschöpfungsnetzwerken einen ganzheitlichen Beziehungsmanagement-Ansatz nicht nur organisatorisch, sondern auch formaljuristisch in Frage stellen. Organisatorisch lässt sich das Problem gut durch die Frage „Wem gehört der Kunde“ beschreiben, auf die es in einem Wertschöpfungsnetzwerk leider keine einfachen Antworten gibt. Formaljuristisch ist die notwendige hohe Integration von Transaktions- und Beziehungsinformationen über Unternehmensgrenzen hinweg fast unmöglich. 


\section{Literatur}

[Alpar et al. 2000]

Alpar, P.; Grob, H.L.; Weimann, P.; Winter, R.: Anwendungsorientierte Wirtschaftsinformatik, 2. Aufl., Braunschweig/Wiesbaden: Vieweg 2000

[Berinato 2001]

Berinato, S.: Building a better bank, in: The Standard, February - March 2001, pp. 82-86, http://www.thestandard.com/grok, March 2001

[Dwyer / Schurr / Oh 1987]

Dwyer, R.F.; Schurr, P.H.; Oh, S.: Developing Buyer-SellerRelationships, in: Journal of Marketing, 51 (1987), pp. 11-27

[Fugmann et al. 1999]

Fugmann T.; Heinrich, B.; Leist, S.; Winter, R.: Banking im Informationszeitalter - Formen und Gestaltungsfragen von Wertschöpfungsnetzwerken im Bankbereich, in: Steiner, M.; Dittmar T.; Willinsky, C. (Hrsg.): Elektronische Dienstleistungswirtschaft und Financial Engineering, Münster: Schüling 1999, S. 237-261

[Gronover / Riempp 2001]

Gronover, S.; Riempp, G.: Kundenprozessorientiertes Multi-ChannelManagement - Management von Mehrkanalsystemen bei Finanzdienstleistern, in: io management, März 2001

[Heinrich 2000]

Heinrich, B.: Dimensionen zur Beschreibung eines Geschäftsmodells für Kreditinstitute im Bereich Privatkunden, Arbeitsbericht BE HSG/CC BAI/01, Version 1.0, Institut für Wirtschaftsinformatik, Universität St. Gallen, Dezember 2000

[Heinrich / Leist 2000]

Heinrich, B.; Leist, S.: Bankenarchitekturen im Informationszeitalter Zur Rolle des Geschäftsmodells, in: Österle, H.; Winter, R. (Hrsg.): Business Engineering, Berlin etc.: Springer 2000, S.141-165

[Leist 2001]

Leist, S.: ???, in: Leist, S.; Winter, R.: Retail Banking im Informationszeitalter, Berlin etc.: Springer, S. ???

[Leist / Winter 1999] 
Leist, S.; Winter, R.: Banking in the information age - Vision, transformation, and design principles, research report BE HSG/FP BAI/01, Institute of Information Management, University of St. Gallen, April 1999

[Leist / Winter 2000]

Leist, S.; Winter, R.: Finanzdienstleistungen im Informationszeitalter Vision, Referenzmodell und Transformation, in: Belz, C.; Bieger, T. (Hrsg.): Dienstleistungskompetenz und innovative Geschäftsmodelle, St. Gallen: Thexis 2000, S. 150-166

[Light 2001]

Light, B.: A review of the issues associated with customer relationship management systems, in: Smithson, S. et al. (eds): ECIS 2001 - 9th European Conference on Information Systems, pp. 1232-1241

[Rogers / Peppers 1994]

Rogers, M.; Peppers, D.: Relationship-Marketing - Planning for share of customers, not market share, in: Sheth, J.N.; Parvatiyar, A. (eds.): Relationship Marketing - Theory, Methods and Applications, Atlanta: Emory University, pp. 391-412

[Peppart 2000]

Peppard, J.: Customer Relationship Management (CRM) in Financial Services, in: European Management Journal, 18 (2000) 3, June, pp. 312327

[Peter / Schneider 1994]

Peter, S.I.; Schneider, W.: Strategiefaktor Kundennähe - Vom Transaktionsdenken zum Relationship Management, in: Marktforschung \& Management, 36 (1994) 1, S. 7-11

[Petzel 2001]

Petzel, E.: Systemarchitekturen für ein erfolgreiches eCRM, in: Bartmann, D.; deMarco, M. (Hrsg.): Proc. Second International Conference on Innovation in the Banking Industry, München, September 2001

[Reisinger 2001]

Reisinger, T.: CRM at Deutsche Bank Private Banking, Vortrag am 5. Data-Warehouse-Forum St. Gallen, 18. Juni 2001, http://www.dwhforum.iwi.unisg.ch

[Schmid / Erni-Grüter / Bach 2001]

Schmid, R.; Erni-Grüter, S.; Bach, V.: Prozessportale als umfassende Kundenschnittstelle, in: io management, März 2001 
[Schmid / Bach / Österle 2001]

Schmid, R.E.; Bach, V.; Österle, H.: Mit Customer Relationship Management zum Prozessportal, in: Bach, V.; Österle, H. (Hrsg.): Customer Relationship Management in der Praxis, Berlin etc.: Springer 2000, S.355

[Schulze 2000a]

Schulze, J.: Prozessorientierte Einführungsmethode für das Customer Relationship Management, Dissertation Nr. 2432, Universität St. Gallen 2000

[Schulze 2000b]

Schulze, J.: Methodische Einführung des Customer Relationship Managements, in: Bach, V.; Österle, H. (Hrsg.): Customer Relationship Management in der Praxis, Berlin etc.: Springer 2000, S.57-84

[Seeger 2001]

Seeger, S.: What Determines the Winners Among Europe's Retail Banks? How CRM Can Provide the Silver Bullet for the Future - A Study of KPMG Consulting and Goldman Sachs, in: Buhl, H.U.; Kreyer, N.; Steck, W. (Hrsg.): e-Finance - Innovative Problemlösungen für Informationssysteme in der Finanzwirtschaft, Berlin etc.: Springer 2001, S.87107

[Sparkassenzeitung 2001]

Auf- und Ausbau der Online-Vertriebswege, Beilage, Die Sparkassenzeitung, Juni 2001

[Swift 2000]

Swift, R.S.: CRM - Creating New Customer Value and High Return On Investment, Presentation at Northwestern University's Kellogg Graduate School of Management, Executive Education, 18.09.2000

[Swift 2001]

Swift, R.S.: Accelerating Customer Relationships - Using CRM and Relationship Technologies, Upper Saddle River: Prentice Hall PTR 2001

[Systor 2001]

Systor AG: En@bling CRM, COR“E e.Business/CRM v1.0, Zürich/Köln 2001

[Williams 2000]

Williams, C.: Customer Relationship Management - Global Architecture, National Australia Bank, June 2000, http://www.national.co.au 
[Winter 2001a]

Winter, R.: Zur Positionierung und Weiterentwicklung des Data Warehousing in der betrieblichen Applikationsarchitektur, in: Schmidt, $\mathrm{H}$. (Hrsg.): Modellierung betrieblicher Informationssysteme (Proc. der MobIS-Fachtagung 2000), Rundbrief der GI-Fachgruppe 5.10, Bd. 7 (2000), Nr. 1, S. 23-38

[Winter 2001b]

Winter, R.: Mass Customization and Beyond - Evolution of Customer Centricity in Financial Services, to appear in: Rautenstrauch, C.; Turowski, K.; Seelmann-Eggebert, R. (eds.): Mass Customization in Progress - Information Systems and Management Principals, Berlin etc.: Springer 2001 